\title{
The association between the timing of energy intake from protein and the risk of overweight and obesity among Saudi female university students
}

\author{
E. Alamri \\ Department of Nutrition and Food Science, University of Tabuk, Saudi Arabi
}

The timing of energy intake plays an important role in weight gain and weight maintenance ${ }^{(1)}$. The literature showed inconsistent findings between the timing of energy intake from protein and weight gain ${ }^{(2)}$. Therefore, the present study aims to investigate the relationship between the timing of energy intake from protein and obesity, as it is still unclear among the Saudi population.

A cross-sectional study was conducted between September 2018 and December 2018. In total, 450 Saudi female students (normal weight were 252 students, obese were 198 students) from the University of Tabuk, Saudi Arabia, were recruited. A four-day dietary record was used to estimate the total energy intake per day and per meal. Portion size of food consumed was estimated by using spoons, cups, serving plates and glasses. The average intake of energy was calculated based on the energy consumed in 4 consecutive days, including weekends. Height and weight were measured at a private place on the university campus according to WHO protocol to calculate BMI. Data were analysed using IBM SPSS Statistics for Windows, version 23 (IBM Corp., Armonk, N.Y., USA). Pearson's correlation coefficients were used to find association between obesity and energy intake. A t test for independent samples was used to compare the differences between the groups.

The study showed that mean of BMI of the obese group was $33 \pm 4.6\left(\mathrm{~kg} / \mathrm{m}^{2}\right)$ and mean of BMI of normal weight group was $21 \pm$ $1.4\left(\mathrm{~kg} / \mathrm{m}^{2}\right)$. There were a significant difference $(\mathrm{P}<0.05)$ in total energy intake per day between the obese group $(2358 \pm 65 \mathrm{kcal})$ and the normal weight group $(1765 \pm 53 \mathrm{kcal})$. It was found a significant negative association between obesity and energy intake from protein at breakfast $(\mathrm{r}=-0.70, \mathrm{P}<0.05)$ and mid-morning $(\mathrm{r}=-0.53, \mathrm{P}<0.05)$. There was no significant difference in the total percentage of energy from protein per day between the obese $(13 \%)$ and normal weight groups $(14 \%)$. However, normal weight groups showed a higher percentage of protein intake in breakfast (4\%) compared to (1\%) among obese group.

In conclusion, the present study showed that greater energy intake from protein in the morning and mid-morning was correlated with a lower risk of overweight and obesity. In addition, the total percentage of energy per day from protein was not different in individuals with overweight or obesity, as compered to individuals with normal weight, apart from breakfast.

1. Garaulet M \& Gómez-Abellán P (2014). Physiol Behav 134, 44-50.

2. Tansey R \& Donald H (2016). P Nutr Soc 75, OCE1 